Annuaire suisse de politique de développement

\title{
Gestion des flux migratoires : réflexions sur la politique française de codéveloppement
}

Jérôme Audran

\section{(2) OpenEdition}

12 Journals

Édition électronique

URL : http://journals.openedition.org/aspd/187

DOI : 10.4000/aspd. 187

ISSN : 1663-9669

Éditeur

Institut de hautes études internationales et du développement

\section{Édition imprimée}

Date de publication : 1 décembre 2008

Pagination : 101-113

ISBN : 978-2-940415-07-6

ISSN : $1660-5934$

\section{Référence électronique}

Jérôme Audran, «Gestion des flux migratoires : réflexions sur la politique française de codéveloppement », Annuaire suisse de politique de développement [En ligne], 27-2 | 2008, mis en ligne le 22 mars 2010, consulté le 08 septembre 2020. URL : http://journals.openedition.org/aspd/187 ; DOI : https://doi.org/10.4000/aspd.187 


\title{
Gestion des flux migratoires: réflexions sur la politique française de codéveloppement
}

\author{
Jérôme Audran*
}

L

a France, comme la plupart des pays développés, a vu sa politique migratoire évoluer au cours des dernières décennies. Traditionnellement ancrée dans des préoccupations sécuritaires, celle-ci s'est peu à peu ouverte à la problématique du développement. Cette nouvelle orientation fait de l'aide publique au développement (APD) un outil d'action visant à atténuer les pressions migratoires allant du Sud vers le Nord. L'APD est ainsi instrumentalisée dans le but de maîtriser avec une plus grande efficacité les flux migratoires. Une telle évolution marque un renouveau de la politique d'immigration française. En effet, après que celle-ci a profondément échoué dans sa tentative d'endiguer l'immigration clandestine via une approche purement coercitive, le développement des pays sources d'immigration s'impose progressivement comme un objectif indispensable pour combattre durablement les flux de migration illégaux ${ }^{1}$. A la stratégie initiale de répression s'ajoute ainsi une stratégie de prévention.

La création récente (mai 2007) en France du Ministère de l'immigration, de l'intégration, de l'identité nationale et du codéveloppement est l'événement le plus significatif de cette instrumentalisation faite de l'APD. La présence du codéveloppement dans l'intitulé de ce nouveau ministère ne relève pas du hasard. Elle est le signe d'une volonté forte du nouveau président de la République de donner à l'aide au développement une nouvelle orientation et une nouvelle dimension ${ }^{2}$. A travers sa participation «à la définition et à la mise en œuvre des politiques de coopération et d'aide au développement qui concourent au contrôle des migrations $»^{3}$, cette nouvelle structure étatique fait non seulement ressurgir l'idée selon laquelle les concepts de développement et de migration sont étroitement corrélés mais marque aussi une insertion idéologique de la sphère du développement dans l'enjeu croissant que représente la migration.

Cette insertion trouve sa source dans une idée très répandue dans les cercles politiques, à savoir que la pauvreté est la cause profonde des migrations internationales ${ }^{4}$. Pour Brice Hortefeux, qui est à la tête du nouveau ministère, le mécanisme des migrations est connu ${ }^{5}$. La pression de l'immigration, qui s'exerce sur

* Diplômé (master en affaires internationales) de l'Institut de hautes études internationales et du développement et analyste en microfinance à Symbiotics, Genève.

1 H. de Haas, Turning the Tide? Why «Development Instead of Migration» Policies are Bound to Fail, Oxford, International Migration Institute, University of Oxford, 2006.

2 B. Hortefeux, «Le codéveloppement, un enjeu majeur pour la France», Les Echos, 31 août 2007.

3 Décret n 2007-999 du 31 mai 2007 relatif aux attributions du ministre de l'Immigration, de l'Intégration, de l'Identité nationale et du Codéveloppement, Journal officiel de la République française, $\mathrm{n}^{\mathrm{o}} 125,1^{\mathrm{er}}$ juin, p. 9964 , texte $\mathrm{n}^{\mathrm{o}} 11$.

4 P. Stalker, «Migration Trends and Migration Policy in Europe», International Migration, vol. 40, $\mathrm{n}^{\mathrm{o}}$ 5, pp. 151-179.

5 B. Hortefeux, op cit. 
le Nord, se nourrit essentiellement des déséquilibres du Sud. Aujourd'hui, le continent africain représente à lui seul $65 \%$ des flux migratoires réguliers vers la France. C'est donc en s'attaquant au problème à son origine, c'est-à-dire au sous-développement, qu'il sera possible de limiter l'immigration illégale. Autrement dit, il est question que la France encourage «le développement des pays d'origine en prenant en compte, mieux que par le passé, la question des flux migratoires $»^{6}$. Brice Hortefeux entend ainsi participer activement à la définition et à la mise en œuvre d'une politique d'aide au développement orientée vers les pays sources d'immigration. Son ambition est claire: faire de la France un exemple en Europe en imaginant et en mettant en œuvre des outils efficaces d'APD, impliquant les migrants et leur permettant de contribuer au développement de leur pays d'origine, afin de «permettre aux ressortissants des pays d'émigration de mieux vivre chez eux plutôt que de survivre ailleurs $»^{7}$.

La politique d'immigration française est donc désormais indissociable de la sphère du développement. Ce schéma se reproduit par ailleurs dans d'autres pays développés ou à l'échelle de l'Union européenne, notamment dans le cadre de son programme $\mathrm{MEDA}^{8}$, qui cherche à «briser le cercle faible croissance, chômage, pauvreté et migration». Dans ce contexte de rapprochement entre coopération au développement et politique migratoire, plusieurs questions se posent naturellement sur le bien-fondé d'une telle démarche. Le lien établi par la politique gouvernementale française en matière d'émigration entre développement des pays d'origine et maîtrise des flux migratoires est-il valide ? Le développement, ou la réduction de la pauvreté, permet-il réellement de diminuer les pressions migratoires? Ne serait-il pas plutôt judicieux de déconnecter le traitement de ces deux questions que constituent d'une part le mouvement des migrations internationales et d'autre part le développement des pays d'origine? Afin de répondre à ces questions, l'examen de plusieurs points s'impose. Dans un premier temps, il convient de décrire l'historique du concept de codéveloppement pour comprendre la signification qu'on lui attribue, ses objectifs et ses enjeux, et voir comment il s'est progressivement institutionnalisé au plus haut sommet de l'Etat français. Une analyse des divers instruments utilisés pour mettre en pratique cette politique de codéveloppement s'avère ensuite indispensable avant de mettre finalement en exergue la pertinence, les côtés positifs et négatifs et les risques de l'instrumentalisation faite de l'APD pour lutter contre l'immigration illégale.

\section{Historique de la notion de codéveloppement}

Le codéveloppement est un concept relativement récent. C'est la coopération française qui l'a forgé et qui a conduit les expériences les plus importantes dans ce domaine. Il a en premier lieu été mis à l'honneur au début des années 1980 avec l'arrivée de la gauche au pouvoir. L'objectif était alors très ambitieux:

6 Discours de Brice Hortefeux lors des «Journées de la coopération internationale et du développement», le 18 juillet $2007,<$ http://www.immigration.gouv.fr/article.php?id_article=85>.

B. Hortefeux, «Le codéveloppement, un enjeu majeur pour la France», op. cit.

8 Le programme MEDA vise à mettre en œuvre les mesures de coopération destinées à aider les pays tiers méditerranéens à procéder à des réformes de leurs structures économiques et sociales et à atténuer les effets du développement économique sur le plan social et environnemental (<http://europa.eu>). 
organiser un véritable codéveloppement de la France et de quelques pays émergents désignés comme partenaires privilégiés en mettant en synergie les complémentarités de la France et de ces pays (savoir-faire et haute technologie d'un côté, main-d'œuvre et ressources naturelles de l'autre). La politique de coopération française visait en d'autres termes à négocier avec les Etats du Sud les modalités de la redistribution équitable des richesses mondiales. Utopie généreuse, comme le souligne Christophe Daum ${ }^{9}$, que la realpolitik a rapidement réduite à peu de chose, le fossé Nord-Sud, loin de se combler, s'étant au contraire fortement aggravé.

A l'époque, il n'était pas question de lier la notion de codéveloppement aux phénomènes migratoires, d'une part parce que l'APD concernait des pays liés à la France par des accords bilatéraux spécifiques et ne s'intéressait pas aux mobilités internationales, mais surtout parce que les pays industrialisés, dont la France, n'étaient pas alors préoccupés par le contrôle des flux migratoires, ne se sentant pas menacés par la migration Sud-Nord. Depuis, l'immigration est devenue un enjeu central de politique intérieure, focalisé non plus uniquement sur l'intégration des immigrés dans la société d'accueil, mais aussi sur les nouveaux arrivants, avec les mouvements sociaux de sans-papiers de 1997. Dans ce contexte, le codéveloppement est apparu comme une réponse appropriée, traitant à la fois les problématiques de l'immigration et du développement. $\mathrm{Au}$ début des années 1990, des expériences ont ainsi été tentées dans la vallée du fleuve Sénégal (ouest du Mali, nord du Sénégal et sud de la Mauritanie), expériences qui ont mené à la définition du codéveloppement dans son acception actuelle. Il s'agissait de proposer des aides financières et un accompagnement par une organisation non gouvernementale aux migrants disposés à rentrer chez eux pour y créer une petite activité économique.

La notion de codéveloppement, au sens où nous l'entendons à présent, a été explicitement formulée en 1998, à l'initiative de Jean-Pierre Chevènement, alors ministre de l'Intérieur. Elle était aussi ambitieuse, mais différente, de celle du début des années 1980. Il s'agissait, avec des objectifs déjà proches de ceux aujourd'hui poursuivis, de valoriser les capacités intellectuelles et financières des migrants au service de leur pays d'origine. Dans la pratique, les actions conduites se sont néanmoins situées dans la continuité des expériences d'aides au retour des années précédentes et se sont en définitive limitées à des aides au retour améliorées. Cette focalisation sur le retour s'est en outre accompagnée de la volonté implicite que l'octroi de crédits pour des actions de codéveloppement soit conditionné par l'obtention de résultats tangibles en matière de maîtrise de l'immigration clandestine. L'ambition des objectifs affichés pour les retours (25000) a inquiété les partenaires sollicités pour la réalisation d'expériences pilotes (Mali, Sénégal, Maroc et Comores) et suscité de vives réticences de leur part, voire même parfois des réactions de rejet ${ }^{10}$.

Dans le discours politique, le terme de codéveloppement recouvre aujourd'hui toute action de coopération en rapport avec les populations immigrées et le

9 C. Daum, «Le codéveloppement, grandeur et décadence d'une aspiration généreuse », Revue internationale et stratégique, $\mathrm{n}^{\circ} 4$ (68), 2007, pp. 49-59.

10 J. Godfrain et R. Cazenave, Rapport sur le codéveloppement, rapport envoyé au Ministère des affaires étrangères de la France, janvier 2007, <http://www.richardcazenave.com/media/Rapport_ codeveloppement.pdf>. 
développement des pays d'origine ${ }^{11}$. Cette définition, relativement large, a été régulièrement reprise dans des termes similaires par les gouvernements successifs. Brigitte Girardin, ancienne ministre déléguée à la Coopération, précisait ainsi en décembre 2006, à 1'occasion de son déplacement au Mali : «S'agissant du concept même de codéveloppement, nous le définissons très simplement comme toute action d'aide au développement à laquelle participent des migrants vivant en France. ${ }^{12}$ Le concept peut aussi bien concerner les immigrés qui désirent retourner dans leur pays pour y créer une activité que ceux - hommes d'affaires, universitaires, médecins et ingénieurs - qui, tout en étant durablement établis en France, sont disposés soit à investir dans leur pays d'origine pour y réaliser des activités productives et/ou des projets sociaux (écoles, dispensaires, etc.), soit à lui faire profiter de leurs compétences, de leurs connaissances et de leurs réseaux de relations ${ }^{13}$.

Même s'il est à présent formellement déconnecté de l'objectif de maîtrise des flux migratoires, le concept de codéveloppement suppose toujours qu'il contribuera à freiner les migrations. C'est ce que l'on constate en pratique du moins. L'idée est que le progrès du développement dans les pays du Sud, engendré par les émigrés, estompera les pressions migratoires en améliorant les conditions de vie et en incitant ainsi les candidats à la migration à rester chez eux plutôt qu'à partir à l'étranger. En d'autres termes, les immigrés d'aujourd'hui contribueraient à la hausse du niveau de vie de leur pays d'origine pour permettre de diminuer les flux migratoires de demain. Par ailleurs, le codéveloppement se matérialise par une instrumentalisation de l'APD à des fins de gestion des flux migratoires. La France octroie en effet une partie de son APD sous conditions, selon l'efficacité de la politique migratoire des pays en développement (PED). Au-delà du simple objectif de développement des pays d'origine généralement annoncé par le gouvernement, la notion de codéveloppement garde donc une forte connotation politique étroitement liée à l'ambition de maîtriser les flux migratoires.

Au niveau institutionnel, la création du nouveau Ministère de l'immigration institutionnalise au plus haut sommet de l'Etat l'intérêt porté aux questions articulant les problématiques de développement et de migration. C'est la première fois qu'un ministère de plein exercice est exclusivement chargé de la politique d'immigration, avec comme attributions, outre celles indiquées dans son intitulé, l'asile et l'accès à la nationalité ${ }^{14}$. Une telle attention attachée à l'immigration démontre son importance en termes d'enjeux électoraux, enjeux largement mis en avant par Nicolas Sarkozy lors des élections présidentielles de 2007. Le nouveau ministère participe notamment à l'élaboration et à la mise en pratique des politiques d'aide et de coopération au développement qui contribuent au contrôle des migrations ${ }^{15}$. En plus de valoriser la contribution des migrants au

11 S. Naïr, Rapport de bilan et d'orientation sur la politique de codéveloppement liée aux flux migratoires, Paris, Mission interministérielle «migrations/codéveloppement», Ministère des affaires étrangères, 1997.

12 Discours de la ministre déléguée à la Coopération, au Développement et à la Francophonie, $M^{m e}$ Brigitte Girardin, Forum national des acteurs du codéveloppement, Paris, 18 décembre 2006.

13 Ministère des affaires étrangères (MEA), Le codéveloppement: présentation générale, Paris, MEA, 26 janvier 2005.

14 Décret $n^{\circ}$ 2007-999 du 31 mai 2007, op. cit.

15 Ibid. 
développement de leur pays d'origine, il s'agit donc d'utiliser l'APD pour mieux contrôler les flux migratoires. Le ministère est doté d'importants moyens, ce qui indique que l'on se situe bien dans l'action et non plus dans une déclaration générale d'intention. Un certain nombre de directions et d'agences ont été placées sous l'autorité du nouveau ministère: l'Agence nationale de l'accueil des étrangers et des migrations, la Police des frontières, la Sous-direction de la circulation des étrangers, etc. D'autres, telle que la Direction générale de la coopération internationale au développement (DGCID), sous tutelle des Affaires étrangères, sont placées à la disposition du Ministère de l'immigration pour ce qui relève de l'exécution des missions de ce dernier. Autrement dit, la DGCID se doit de participer à tout projet de développement, à l'initiative du nouveau ministère, impliquant les migrants et/ou ayant pour objectif de maîtriser les flux migratoires. S'agissant du budget attribué au ministère, qui s'élève à 639 millions d'euros en crédits de paiement en 2008, il se répartit par grandes politiques publiques, avec 49,9\% dédiés à l'asile, 29,6\% à l'accueil et à l'intégration des étrangers titulaires d'un titre de séjour, $13 \%$ à la politique d'immigration, 7,2\% aux dépenses d'état major, 4,5\% au codéveloppement et $0,2 \%$ aux naturalisations ${ }^{16}$. Si cet organigramme institutionnel et ces dotations relèvent en partie d'une réorganisation de moyens affectés auparavant à d'autres ministères, leur mise en cohérence témoigne d'une logique pragmatique au service de choix politiques identifiables. Un examen attentif de la façon dont se décomposent ces différents chapitres révèle en effet que nous sommes dans une logique fortement imprégnée par l'objectif de contrôle migratoire ${ }^{17}$.

\section{Typologie des actions de codéveloppement}

La politique française de codéveloppement, qui se matérialise par l'attribution d'aides bilatérales et par la participation des immigrés aux projets entrepris, est mise en œuvre via divers instruments qui se regroupent en quatre grands champs d'action: $1^{\circ}$ les mesures visant à mobiliser les compétences des élites de la diaspora, $2^{\circ}$ les projets d'aménagement local, auxquels les associations de migrants sont très attachées, $3^{\circ}$ les aides à la réinsertion (locution préférée à celle d' «aide au retour», plus péjorative, autrefois utilisée), et $4^{\circ}$ les investissements productifs dans les pays à l'origine des flux migratoires.

Afin de créer une cohésion entre ces divers outils et gérer la migration de façon coordonnée, la France établit sur une base bilatérale des accords-cadres de gestion concertée des flux migratoires. Ces accords, qui lient la question du développement à celle des migrations Sud-Nord, entendent poser les bases d'un nouveau partenariat entre la France et les PED $^{18}$. Le codéveloppement se situe à la confluence de trois problématiques essentielles: les migrations internationales, la mondialisation et le développement. Et les défis qui se posent dans ces trois

16 Le programme «codéveloppement» est doté de 29 millions d'euros en crédits de paiement et de 60 millions d'euros en autorisations d'engagement. Budget 2008 du Ministère de l'immigration, de l'intégration, de l'identité nationale et du codéveloppement, <http://www.anaem.fr>.

17 C. Daum, op. cit.

18 R. Munnich, Les accords de codéveloppement, un nouvel instrument de coopération avec le Sud, Paris, Haut Conseil de la coopération internationale, novembre 2007, <http://www.hcci.gouv.fr/ lecture/fiches/accord-de-co-developpement.html>. 
domaines concernent aussi bien les pays du Nord que ceux du Sud. Des solutions viables ne pourront donc être trouvées que dans le cadre d'un dialogue tripartite entre les Etats concernés et les migrants. Cela suppose que la maitrise des flux migratoires ne peut reposer exclusivement sur l'application d'une législation et d'une réglementation nationales définissant les conditions d'entrée et de séjour des ressortissants étrangers; elle doit également faire appel à la négociation internationale et intégrer une vision nouvelle de l'APD. L'objectif des accords-cadres est en ce sens d'organiser la coopération Nord-Sud en partenariat avec les PED de façon à gérer avec eux la migration légale, la lutte contre l'immigration irrégulière et les actions de codéveloppement et à faire en sorte que la migration profite à la fois aux pays sources et aux pays receveurs. La participation des migrants et de leurs associations aux actions d'aide au développement peut par ailleurs contribuer à leur meilleure intégration dans la société française et à ce qu'ils gardent des liens étroits avec leur terre natale.

De tels accords sont censés constituer une base de négociation évolutive sur laquelle les pays d'origine font part de leurs besoins à la France tout en s'engageant en contrepartie à lutter contre la migration illégale. Si l'objectif de développer les pays sources d'émigration est poursuivi à travers ces accords, ces derniers visent donc aussi en grande partie à lutter contre l'immigration clandestine. Comme le souligne Brice Hortefeux: «Les pays d'origine feront part [à la France] de leurs besoins et s'engageront à [ses] côtés contre l'immigration illégale, en échange de quoi [la France] accueillera un certain nombre de leurs ressortissants et formera leurs élites. ${ }^{19}$ Ceci explique pourquoi les accords-cadres incluent un volet de réadmission des migrants clandestins dans leur pays d'origine et comportent généralement un volet codéveloppement mesuré par le second indicateur (c'est-à-dire le nombre de réadmissions). L'enjeu est explicite: il s'agit de monnayer la bonne volonté des Etats pourvoyeurs d'émigration dans le contrôle de celle-ci ${ }^{20}$.

L'instrumentalisation de l'APD par le gouvernement français se fait donc de deux manières visant à maîtriser les flux migratoires, l'une par le développement et l'autre par la conditionnalité de l'aide. La première consiste à financer des programmes de développement dans des régions à fortes pressions migratoires de façon à améliorer les conditions de vie des populations et à les inciter ainsi à rester (ou à rentrer) chez elles plutôt qu'à opter pour la migration. La seconde vise à octroyer l'APD aux pays du Sud selon des critères de lutte contre la migration clandestine et l'efficacité de leur politique en la matière.

\section{Le codéveloppement: penser la migration en termes d'intérêts partagés}

Dans ce contexte d'instrumentalisation de l'APD par la France, il convient de s'interroger sur la pertinence, les bénéfices et les risques d'une telle politique.

Tel qu'il est mis en avant par le gouvernement français, le codéveloppement constitue tout d'abord un moyen de penser la migration en termes d'intérêts partagés. En intégrant les émigrés dans la coopération Nord-Sud, à travers la mise à contribution de leurs investissements, connaissances et savoir-faire, les PED

19 B. Hortefeux, «Le codéveloppement, un enjeu majeur pour la France», op. cit.

20 C. Daum, op. cit. 
bénéficient de ressources financières et humaines inestimables. Dans le passé, l'investissement des associations de migrants a d'ailleurs engendré de grands bouleversements dans leurs localités d'origine. L'APD attribuée dans le cadre du codéveloppement a aussi permis de développer des programmes de développement d'envergure fortement bénéfiques aux populations locales. Pour les pays du Nord, le codéveloppement s'inscrit dans leur politique d'immigration choisie. L'instrumentalisation de l'aide est un moyen de lutter contre l'immigration clandestine et d'atténuer les pressions migratoires. Le codéveloppement répond donc à l'impératif de solidarité qui est au cœur du pacte républicain français mais s'inscrit aussi dans l'agenda politique du gouvernement (objectif de répondre aux préoccupations du peuple liées aux questions migratoires). Cela reflète la stratégie de coopération au développement de la France, qui vise à promouvoir à la fois ses intérêts et ceux de ses pays partenaires.

En liant l'APD et la lutte contre la migration clandestine, le gouvernement français entend donc réduire la pauvreté au Sud via le codéveloppement tout en permettant à la France de lutter plus efficacement contre les flux migratoires illégaux. C'est pour lui un domaine d'action politique capable de renforcer la coopération Nord-Sud et celle entre Etat et société civile. Le codéveloppement offre en ce sens, tout au moins en principe, un cadre adéquat de concertation et de coopération interétatiques dans lequel toutes les problématiques liées aux flux migratoires peuvent être traitées de façon coordonnée. Lier les questions de coopération au développement et de migration peut aussi avoir l'avantage de rappeler aux pays occidentaux que les différents continents ont un avenir interdépendant et qu'ils subiront de toute manière les conséquences du manque d'efforts réalisés pour niveler un tant soit peu les conditions de vie de par le monde. Si l'objectif de développement des pays d'origine est sincèrement poursuivi pour lutter contre la migration, cela poussera donc les pays du Nord à remettre en cause leurs politiques et à se tourner davantage vers les pays du Sud pour mieux identifier leurs besoins de développement et mieux y répondre. Et si l'on suppose que la réduction de la pauvreté contribuera à freiner les migrations, comme c'est le cas dans les discours politiques, toute stratégie politique cohérente visant à mieux maîtriser la migration passera inévitablement par une hausse de l'APD et une redéfinition par les pays du Nord de leurs politiques de coopération au développement.

A travers ses cadres de gestion concertée des flux migratoires et sa politique de codéveloppement, la France dispose donc d'un précieux outil pour impliquer avec plus d'enthousiasme les pays africains et la société civile dans la lutte contre l'immigration illégale ${ }^{21}$. Renvoyer un immigré clandestin en lui accordant un microcrédit pour créer son entreprise est très certainement mieux perçu par les migrants et les gouvernements du Sud (et plus efficace) qu'une simple expulsion. De même, mettre en place des programmes de développement au Sud incite davantage les PED à mieux gérer leurs flux migratoires. Chercher à réduire la migration à travers un but commun - le développement - peut donc renforcer la coopération Nord-Sud. Cependant, encore faut-il que cette coopération soit réellement pensée en termes d'intérêts partagés.

21 C. Tasca, J. Pelletier et B. Barraux, Le codéveloppement à l'essai, Rapport d'information, $\mathrm{n}^{\circ} 417$ (2006-2007), Paris, Sénat, 25 juillet 2007. 
Avant de critiquer la politique française de codéveloppement, il est nécessaire de s'interroger sur son efficacité lorsqu'elle vise à fixer les populations du Sud.

Chercher à réduire les pressions migratoires en aidant les pays du Sud à se développer paraît être une stratégie politique non seulement inefficace mais aussi contradictoire. En soi, l'idée que le codéveloppement contribuerait à freiner les migrations n'est peut-être pas fausse - mais seulement si on accepte de se projeter dans un long horizon temporel et si certaines conditions sont réunies.

Plusieurs travaux de recherche ont démontré que le développement des pays du Sud ralentit les flux migratoires, mais seulement sur un long terme qui dans bien des cas peut se mesurer en siècles et dans un contexte de réduction substantielle des inégalités nationales et internationales dans la répartition des richesses ${ }^{22}$. Selon ces études, commencées au début des années 1970 par Wilbur Zelinski (mobility transition model) puis poursuivies par d'autres chercheurs dont Ralph Rotte, Michael Vogler et Hein de Haas -, le processus de développement que les sociétés traversent mène à un changement en plusieurs étapes de la propension des populations à migrer à l'étranger. Les liens entre développement et migrations internationales diffèrent ainsi selon l'horizon temporel et se schématisent par une courbe en $U$ renversé: dans un premier temps, le développement contribue à accroître la migration puis, après avoir atteint une phase de stabilisation, à la diminuer. Cela est dû au fait que le développement augmente les aspirations et les capacités des ménages à migrer via la dissolution des restrictions financières liées à la migration, la destruction de l'emploi agricole en milieu rural, l'accroissement des inégalités économiques et l'amélioration du capital humain et social. De tels changements contribuent à la migration des populations rurales vers les villes, ce qui alimente les flux migratoires et élargit la possibilité pour les individus de migrer. Ce n'est que dans un second temps, quand les effets combinés de la transition démographique, de l'accroissement de la productivité agricole, du développement économique et de la baisse des inégalités se font sentir, que l'émigration s'épuise et s'inverse quelquefois.

La réponse à la question «le développement permet-il de réduire les flux migratoires?» dépend ainsi de la durée. A long terme, oui, mais à certaines conditions; à court et à moyen terme, non. Tout développement introduit des déséquilibres. Et dans les régions rurales, le bouleversement des rapports sociaux est tel qu'il entraîne un exode à l'origine de flux migratoires internationaux. Les politiques ayant pour objectif de substituer (à court terme) la migration par le développement reposent par conséquent sur une hypothèse fausse selon laquelle les migrants potentiels, s'ils vivent mieux chez eux, écartent de fait l'option de la migration. Cette hypothèse ne tient compte que de la pauvreté absolue et non des inégalités et des profonds changements engendrés par le développement. Le codéveloppement est en fait fort susceptible de provoquer

22 Voir notamment les travaux suivants: R. Rotte and M. Vogler, The Effects of Development on Migration: Theoretical Issues and New Empirical Evidence, IZA Discussion Papers, ${ }^{\circ} 46$, Bonn, Institute for the Study of Labor (IZA). R.H. Adams, Jr. and J. Page, International Migration, Remittances, and Poverty in Developing Countries, World Bank Policy Research Working Paper, $\mathrm{n}^{\circ} 3179$, Washington, DC, World Bank. 
une hausse des flux migratoires du Sud vers le Nord pendant plusieurs décennies; la durée de cette hausse dépend du niveau de développement initial d'un pays et de l'évolution de sa situation économique et sociale. Cela est en particulier vrai pour la France, dont la majorité des immigrés proviennent du continent africain. Lorsqu'on connaît le niveau de pauvreté de l'Afrique, il est clair que son développement constitue avant tout un danger pour la France en matière d'immigration clandestine. En faisant preuve de cynisme, on peut affirmer qu'il est plus efficace de maintenir l'Afrique subsaharienne dans une situation d'extrême pauvreté plutôt que chercher à favoriser son développement. Seule une politique de développement durable du continent africain - généreuse et inscrite sur le long terme - pourra diminuer les pressions migratoires. Mais il n'est pas possible de fixer les populations du Sud sans passer par une phase de pressions migratoires accrues. Croire ou faire croire qu'il est possible de fixer les populations dans l'espace d'un ou quelques mandats politiques via le codéveloppement relève donc de l'imaginaire.

\section{Les limites du codéveloppement}

Au-delà de cette contradiction politique, plusieurs risques et limites doivent être mis en avant pour aider à déceler les enjeux sous-jacents au concept de codéveloppement et à sa mise en pratique en France.

Depuis son origine, le codéveloppement balance fortement entre deux logiques de politique publique: d'une part, appuyer l'engagement des migrants dans le développement de leur pays d'origine et, d'autre part, renforcer les dispositifs de contrôle des frontières et de lutte contre l'immigration clandestine. Dans le premier cas, il s'agit de maîtriser les flux migratoires vers l'Europe en encourageant le développement des pays source d'émigration. L'objectif poursuivi - le développement - est partagé entre les pays du Sud et du Nord même si, pour ces derniers, le développement n'est qu'un moyen pour atteindre un objectif politique prédéterminé: maîtriser les pressions migratoires. Dans le second cas, il s'agit de renforcer la dimension répressive de la politique migratoire en fixant des conditionnalités aux pays bénéficiaires de l'aide. L'APD est ainsi distribuée lorsque certaines politiques conformes aux intérêts de la France sont mises en place et que certains indicateurs de performance (réadmission des migrants clandestins par exemple) sont atteints.

Le codéveloppement apparaît donc comme un instrument ambigu qui sert à la fois de programme de développement et d'accompagnement des politiques sécuritaires de la France. L'intégration des migrants dans la coopération Nord-Sud, et l'instrumentalisation qui est en faite au service d'un développement économique censé répondre au problème migratoire, viennent encore renforcer les ambiguïtés de cette notion fortement politisée. Il existe enfin un décalage apparent entre le discours politique portant sur le codéveloppement et sa mise en pratique effective. En effet, si l'analyse de la mission et du budget attribués au programme de codéveloppement démontre ses orientations relevant du contrôle de l'immigration $^{23}$, les partisans de cette politique persistent à dire qu'il se démarque des approches sécuritaires de l'immigration et que sa vocation première n'est pas

23 C. Daum, op. cit. 
d'endiguer les flux migratoires mais de valoriser les efforts des migrants au service du développement de leur communauté d'origine. Ils insistent ainsi sur les trois points forts (ou positifs) du codéveloppement: faciliter les initiatives des migrants dans leur pays d'origine, favoriser l'investissement des migrants et agir contre la fuite des cerveaux. Les auteurs d'un rapport parlementaire récent pour le Ministère des affaires étrangères signalent ainsi que «le codéveloppement ne peut être compris comme un moyen détourné de renvoyer les migrants dans leur pays d'origine. De même, il n'a pas pour vocation de tarir les flux migratoires $»^{24}$. Pourtant, la réalité est tout autre, avec la fixation de conditionnalités et le ciblage de programmes de développement dans les zones à fortes pressions migratoires.

D'un point de vue politique, cette ambiguïté présente un risque pour la coopération Nord-Sud: celui que les PED rejettent la politique de codéveloppement par peur que leurs intérêts ne soient pas suffisamment pris en compte. Comme l'estime Aminata Dramane Traoré, ancienne ministre de la Culture du Mali, «le codéveloppement peut être résumé dans les circonstances actuelles comme la touche d'humanité sur laquelle comptent les artisans de l'immigration choisie, à la fois pour freiner les départs et pour favoriser le retour de ceux qui se laissent convaincre $\gg^{25}$. Autrement dit, le codéveloppement est perçu comme un instrument déguisé visant à diminuer l'immigration clandestine plutôt qu'à promouvoir le développement des pays au Sud. Pour que le codéveloppement fonctionne bien, il faudrait qu'il soit non seulement plus transparent, mais aussi envisagé en termes d'intérêts partagés.

Par ailleurs, le codéveloppement ne suffit pas à lui seul pour lutter efficacement contre les pressions migratoires. L'ambition affichée - freiner les migrations via le codéveloppement - est sans rapport avec les faiblesses des moyens apportés et des efforts consentis. Le développement ne pourra freiner les flux migratoires qu'à travers une politique de développement durable s'inscrivant sur le long terme. Certes, comme le met en avant Brice Hortefeux, le budget du codéveloppement est passé de 3 à 60 millions d'euros sous la présidence de Nicolas Sarkozy, ce qui permet de multiplier par vingt les engagements de la France dans ce domaine ${ }^{26}$. Mais ce budget reste très largement insuffisant. Il est de 20 millions inférieur à celui affecté à la Police des frontières et aux reconduites dans les pays d'origine. De plus, il faudra que le gouvernement augmente drastiquement son aide s'il entend freiner la migration clandestine. Elle ne représente que $0,39 \%$ du revenu brut de la France en 2007, ce qui est encore loin de l'objectif de 0,70\% fixé en 2002 lors de la conférence de Monterrey. Enfin, s’il s'agit de freiner les migrations à la source, ce n'est pas seulement une politique de codéveloppement substantielle qu'il faudra mettre en place. S'engager dans cette voie impliquera de redéfinir en profondeur l'APD de manière générale (les moyens qui y sont consacrés et les modalités qui sont appliquées), mais aussi de revoir les politiques commerciales et de résoudre les nombreux conflits en cours, à l'origine de migrations forcées dans les PED. C'est pourquoi il est illu-

24 J. Godfrain et R. Cazenave, op. cit.

25 A.D. Traoré, Migrations africaines et violences: Où est le danger? Où est la vérité ?, Bamako, Forum pour un autre Mali (FORAM), 17 juin 2007, <http://www.co-developpement.org/?p=331>.

26 M. Joannidis, Le codéveloppement pourra-t-il freiner l'immigration?, Radio France Internationale,

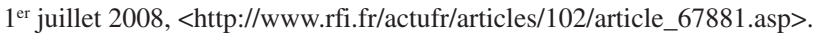


soire de penser que le codéveloppement puisse à lui seul apporter des solutions aux phénomènes migratoires ${ }^{27}$. Ce n'est pas en tout cas pour cette raison qu'il faut s'y intéresser.

Enfin, le fait d'instrumentaliser l'APD à des fins de gestion des flux migratoires présente le risque de la détourner de son objectif initial : celui de réduire la pauvreté. Le souhait actuel de la France, en matière de codéveloppement, est de se doter d'une approche très pragmatique de l'APD qui vise, à travers des actions sectorielles et géographiques, à limiter les flux migratoires. Il s'agit de concentrer l'APD sur les régions sources d'émigration où seront mis en place des projets créateurs d'emplois afin de fixer les populations ${ }^{28}$. Une telle approche entre en contradiction avec la raison d'être de l'APD, qui est de lutter contre la pauvreté, où qu'elle se situe. Alors que les migrants internationaux ne proviennent ni des pays les plus pauvres (la migration émane avant tout des PED à revenu intermédiaire) ni des strates les plus pauvres de ces pays (les migrants se situent en général au-dessus du seuil de pauvreté ${ }^{29}$, l'octroi accru de fonds assignés au développement - déjà fortement limités et mal distribués - sur la base du critère migratoire viendra ainsi accentuer les inégalités socioéconomiques nationales et internationales. Par ailleurs, l'argent affecté à la politique de codéveloppement est directement dégagé du budget de l'APD géré par la DGCID. Certes, les 60 millions d'euros qui constituent le budget du codéveloppement ne représentent qu'une infime partie de l'APD de la France. Mais si cette portion était amenée à s'accroître à l'avenir, il serait crucial que le budget attribué au codéveloppement vienne en complément et non en substitution à celui de l'APD. Une politique d'aide et de coopération au développement doit en premier lieu cibler les pays qui en ont le plus besoin. C'est ainsi qu'il sera possible d'atteindre les Objectifs du Millénaire pour le développement. Si une distinction claire n'est pas faite entre APD et codéveloppement, la politique d'aide française pourrait se détourner de ses objectifs initiaux à mesure que le budget consacré au codéveloppement augmentera.

\section{Conclusions}

Depuis le début des années 1980, les problématiques de développement et de migration se sont progressivement rapprochées dans la sphère politique. Le concept de codéveloppement est ainsi apparu et a progressivement reçu une attention croissante dans un contexte où l'immigration est devenue un enjeu central de politique intérieure. C'est en France que ce concept est né et s'est le plus rapidement institutionnalisé, avec la création du nouveau Ministère de l'immigration. Une telle évolution, qui se matérialise par l'instrumentalisation de l'APD à des fins de gestion des flux migratoires, marque la volonté du gouvernement français de lier les questions de migration et de coopération au développement de façon à maximiser les bienfaits de la migration sur le développement

27 V. Vanhaeverbeke, «Le codéveloppement, un concept qui se cherche encore», Echos du COTA, dossier Quel partenariat entre ONG et migrants ?, no 110, Bruxelles, COTA asbl, mars 2006, pp. 3-5.

28 Allocution de la ministre déléguée à la Coopération, au Développement et à la Francophonie, $M^{m e}$ Brigitte Girardin, Conférence ministérielle euro-africaine sur la migration et le développement, Rabat, 10 juillet 2006.

29 R.H. Adams, Jr. and J. Page, op. cit. 
des pays du Sud dans le but de fixer les populations et de contrôler avec une plus grande efficacité l'immigration clandestine via un système de conditionnalité de l'aide.

Le codéveloppement prend aussi de l'importance dans l'agenda politique international. Le concept est en effet vite sorti de l'Hexagone pour rentrer dans le vocabulaire des institutions internationales, et notamment à l'échelle de l'Union européenne où il a fait son apparition dès le sommet de Tampere en 1999. En 2004, le Parlement européen a ainsi affirmé «regretter que jusqu'à présent les mesures adoptées par le Conseil et les Etats membres pour le contrôle des vagues migratoires aient été des mesures de contrôle répressives plutôt que des mesures positives proactives». Il a par ailleurs rappelé que «les stratégies visant à réduire la pauvreté, à améliorer les conditions de vie et de travail, à créer des emplois et à développer la formation dans les pays d'origine, contribuent à long terme à normaliser les flux migratoires $»^{30}$. Aujourd'hui, à la suite de la présidence française de l'Union européenne, le concept de codéveloppement est au cœur de l'agenda politique. Comme le souligne Brice Hortefeux, «l'Union européenne a trop longtemps ignoré le lien entre l'aide au développement et la lutte contre l'immigration irrégulière ${ }^{31}$. Le gouvernement français, conscient que les initiatives prises par la France dans ce domaine ne doivent pas rester isolées en Europe, se propose ainsi d'approfondir la concertation avec la Commission européenne et les autres Etats membres de l'Union européenne en matière de maîtrise des flux migratoires et de codéveloppement ${ }^{32}$. Cette volonté se matérialise notamment dans le nouveau «Pacte européen sur l'immigration», qui inclut un volet sur le codéveloppement.

Dans ce contexte d'essor du codéveloppement, il est plus que jamais crucial d'analyser sa pertinence, de définir ses contours et de déceler ses avantages et limites en termes d'efficacité. Comme nous l'avons vu à travers le cas de la France, le codéveloppement offre la possibilité de penser la migration en termes d'intérêts partagés et de renforcer ainsi la coopération Nord-Sud. C'est donc une innovation politique majeure. Cependant, il présente certaines limites et incohérences dans sa mise en pratique dont il convient de tenir compte. Premièrement, chercher à réduire les pressions migratoires en aidant les pays du Sud à se développer est une stratégie politique inefficace et fortement contradictoire, tout au moins à court terme. Deuxièmement, malgré les efforts de définition du codéveloppement, la notion reste ambiguë - vacillant entre l'objectif de développement des pays d'origine et celui de lutte contre l'immigration clandestine -, avec le risque pour la France que les pays du Sud et les migrants perçoivent le codéveloppement comme un moyen détourné de promouvoir unilatéralement ses intérêts. Troisièmement, le codéveloppement ne suffit pas à lutter contre les pressions migratoires. Seule une croissance économique rapide, soutenue et égalitaire des PED - alimentée par une hausse des budgets affectés au codéveloppement et à l'APD, par une remise en question des politiques commerciales du Nord et par des efforts de résolution des conflits en cours - pourra durable-

30 Parlement européen, Résolution du Parlement européen sur les liens entre immigration légale et illégale et l'intégration des migrants (2004/2137), 2004.

31 B. Hortefeux, «Le codéveloppement, un enjeu majeur pour la France», op. cit.

32 Conseil des ministres, Les accords de gestion concertée des flux migratoires et de codéveloppement, communiqué du Conseil des ministres, 29 août 2007. 
ment diminuer les flux migratoires illégaux. Enfin, le fait d'instrumentaliser l'APD dans l'objectif de mieux contrôler les flux migratoires risque de détourner la coopération au développement de son objectif initial: combattre la pauvreté.

Le codéveloppement offre donc un cadre de coopération tripartite pouvant renforcer l'impact de la migration sur le développement du Sud et permettre d'améliorer la maîtrise des flux migratoires vers le Nord. Mais pour qu'il atteigne ces objectifs, il est indispensable que les pays du Nord mettent en place une politique d'APD et de codéveloppement ambitieuse, pensent le codéveloppement en termes d'intérêts partagés et fassent une distinction claire entre l'APD classique et celle qui est instrumentalisée pour contrôler les flux migratoires. Sans cela, le codéveloppement pourrait ne pas dépasser le stade de projet politique ambitieux ou même engendrer des effets inverses de ceux qui sont recherchés.

\section{Bibliographie}

Adams, R.H. Jr. and J. Page, International Migration, Remittances, and Poverty in Developing Countries, World Bank Policy Research Working Paper, nº 3179, Washington, DC, World Bank.

Cazenave, R. et J. Godfrain, Rapport sur le codéveloppement, rapport envoyé au Ministère des affaires étrangères de la France, janvier 2007, <http://www.richardcazenave.com/media/Rapport codeveloppement.pdf $>$.

Conseil des ministres, Les accords de gestion concertée des flux migratoires et de codéveloppement, communiqué du Conseil des ministres, 29 août 2007.

Daum, C., «Le codéveloppement, grandeur et décadence d'une aspiration généreuse », Revue internationale et stratégique, $\mathrm{n}^{\circ} 4$ (68), 2007, pp. 49-59.

Décret n 2007-999 du 31 mai 2007 relatif aux attributions du ministre de l'Immigration, de l'Intégration, de l'Identité nationale et du Codéveloppement, Journal officiel de la République française, $\mathrm{n}^{\circ} 125$, $1^{\text {er }}$ juin, p. 9964, texte $n^{\text {o }} 11$.

Guengant, J.-P., «Quel lien entre migrations internationales et développement?», Projet, dossier Migrations et frontières, $\mathrm{n}^{\circ}$ 272, La Plaine Saint-Denis, Centre d'étude et de recherche en action sociale (CERAS), décembre 2002.

Haas, H. de, Turning the Tide? Why «Development Instead of Migration» Policies are Bound to Fail, Oxford, International Migration Institute, University of Oxford, 2006

Hortefeux, B., «Le codéveloppement, un enjeu majeur pour la France», Les Echos, 31 août 2007.

Joannidis, M., Le codéveloppement pourra-t-il freiner l'immigration?, Radio France Internationale,

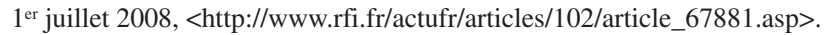

Ministère des affaires étrangères (MEA), Le codéveloppement: présentation générale, Paris, MEA, 26 janvier 2005.

Munnich, R., Les accords de codéveloppement, un nouvel instrument de coopération avec le Sud, Paris, Haut Conseil de la coopération internationale, novembre 2007, <http://www.hcci.gouv.fr/lecture/ fiches/accord-de-co-developpement.html>.

Parlement européen, Résolution du Parlement européen sur les liens entre immigration légale et illégale et l'intégration des migrants (2004/2137), 2004.

Rotte, R. and M. Vogler, The Effects of Development on Migration: Theoretical Issues and New Empirical Evidence, IZA Discussion Papers, n 46, Bonn, Institute for the Study of Labor (IZA).

Stalker, P., «Migration Trends and Migration Policy in Europe», International Migration, vol. 40, $\mathrm{n}^{\circ} 5$, pp. 151-179.

Tasca, C., J. Pelletier et B. Barraux, Le codéveloppement à l'essai, Rapport d'information, nº 417 (20062007), Paris, Sénat, 25 juillet 2007.

Traoré, A.D., Migrations africaines et violences: Où est le danger ? Où est la vérité?, Bamako, Forum pour un autre Mali (FORAM), 17 juin 2007, <http://www.co-developpement.org/?p=331>.

Vanhaeverbeke, V., «Le codéveloppement, un concept qui se cherche encore», Echos du COTA, dossier Quel partenariat entre ONG et migrants?, $\mathrm{n}^{\circ} 110$, Bruxelles, COTA asbl, mars 2006, pp. 3-5. 